\title{
Invasive Candidiasis in Patients with Solid Tumors Treated with Anidulafungin: A Post Hoc Analysis of Efficacy and Safety of Six Pooled Studies
}

\author{
Francesco Giuseppe De Rosa ${ }^{1}\left[\right.$ - Alessandro Busca ${ }^{2} \cdot$ Maria Rita Capparella ${ }^{3} \cdot$ Jean Li Yan ${ }^{4} \cdot$ Jalal A. Aram $^{4}$
}

Accepted: 6 March 2021 / Published online: 23 April 2021

(c) The Author(s) 2021

\begin{abstract}
Background Solid tumors are a common predisposing factor for invasive candidiasis (IC) or candidemia due to IC. Objectives Post hoc analysis of patient-level efficacy and safety data from six studies of anidulafungin (with similar protocols/ endpoints) in adults with IC/candidemia summarized by past or recent diagnosis of solid tumors.

Patients/methods Patients received a single intravenous (IV) dose of anidulafungin $200 \mathrm{mg}$, followed by $100 \mathrm{mg}$ once daily. After $\geq 5$ to $\geq 10$ days of IV treatment, switch to oral voriconazole/fluconazole was permitted in all but one study. Time of solid tumor diagnosis was defined as past, $\geq 6$; and recent, $<6$ months prior to study entry. Primary endpoint: global response of success (GRS) rate at the end of IV therapy (EOIVT). Secondary endpoints included the GRS rate at the end of all therapy (EOT), all-cause mortality, and safety.

Results The GRS rate in the overall population was $73.4 \%$ at EOIVT and $65.5 \%$ at EOT. Past or recent solid tumor diagnosis did not affect GRS at EOIVT or EOT (past: $75.5 \%$ and $71.4 \%$; recent: $72.2 \%$ and $62.2 \%$, respectively). All-cause mortality was $14.4 \%$ on day 14 and $20.1 \%$ at day 28 . Most treatment-emergent adverse events were mild/moderate in severity $(81.6 \%)$. Conclusions Treatment of IC was effective regardless of the time of solid tumor diagnosis.

Trial Registration Data were pooled from six studies: NCT00496197 (first posted on ClinicalTrials.gov on July 4, 2007); NCT00548262 (first posted on ClinicalTrials.gov on October 23, 2007); NCT00537329 (first posted on ClinicalTrials.gov on October 1, 2007); NCT00689338 (first posted on ClinicalTrials.gov on June 3, 2008); NCT00806351 (first posted on ClinicalTrials.gov on December 10, 2008); NCT00805740 (first posted on ClinicalTrials.gov on December 10, 2008).
\end{abstract}

\section{Plain Language Summary}

Patients with solid tumor cancers (cancer of internal organs) have increased risk of fungal infections that can spread in the body through the blood. Infection with Candida species, known as invasive candidiasis (IC) (Candida invades the body in places normally free from germs) or candidemia (Candida infection in the blood), can cause severe illness and/or death. Anidulafungin is an antifungal drug recommended to treat IC/candidemia. This post hoc analysis looked at how effective and safe anidulafungin was in adult patients with IC/candidemia with 'recent' or 'past' history of solid tumors. The analysis included patients diagnosed with cancer less than 6 months before (recent history) or more than 6 months before (past history) they first received anidulafungin. Patients received anidulafungin by injection (intravenously [IV]) into the veins and, for continued treatment, were able to take a different antifungal drug orally. Of 539 patients from six studies, 139 had confirmed IC/candidemia and a history of solid tumors. Approximately 7 out of $10(72 \%)$ patients were cured or no longer had signs of Candida infection at the end of IV anidulafungin treatment. Results were similar in patients with past or recent diagnosis of solid tumors. Treatment side effects reported in approximately 8 out of $10(82 \%)$ patients were mild-to-moderate in severity. This analysis suggests anidulafungin was well tolerated and effective at treating IC/candidemia in patients with solid tumors, whether diagnosed recently or in the past.

Francesco Giuseppe De Rosa

francescogiuseppe.derosa@unito.it

1 Department of Medical Sciences, University of Turin, Corso Bramante 88, 10126 Turin, Italy
2 Department of Oncology and Hematology, Stem Cell Transplant Centre, Turin, Italy

3 Pfizer PIO, Paris, France

4 Pfizer Inc, New York, NY, USA 
Digital Features for this article can be found at https://figshare. $\mathrm{com} / \mathrm{s} / 5 \mathrm{e} 036327 \mathrm{f} 480 \mathrm{a} 030 \mathrm{a} 490$.

\section{Key Points}

The primary measure of efficacy used in this study (global response of success rate) at the end of IV treatment with anidulafungin in patients with invasive candidiasis/candidemia and solid tumors was comparable to that of a representative sample of patients with IC/ candidemia.

In this analysis, treatment with anidulafungin was effective irrespective of a past or recent diagnosis of solid tumors.
Echinocandins, including anidulafungin, target Candida species by inhibiting beta-(1,3)-D-glucan synthase, essential for the synthesis of fungal cell wall glucan [12]. Anidulafungin is approved in the USA and Europe for the treatment of IC/candidemia $[15,16]$. Previous pooled analyses of anidulafungin studies have demonstrated effectiveness and tolerability in patients with IC/candidemia, intra-abdominal candidiasis, neutropenia, and infections caused by $C$. parapsilosis and C. krusei [17-19].

To address the relative scarcity of data on the treatment of patients with invasive fungal infections and malignancies, and to explore the temporal effect of tumor diagnosis on the risk of fungal infection, this post hoc analysis investigated whether the efficacy and safety of anidulafungin treatment of patients with IC/candidemia and solid tumors varied when summarized by past or recent diagnosis of solid tumor diagnosis.

\section{Patients and Methods}

\section{Introduction}

Patients with cancer, particularly solid tumors and hematologic malignancies, are at increased risk of invasive candidiasis (IC) [1] or candidemia due to IC [2]. In an analysis of European multi-institutional surveys of patients with candidemia, an underlying pathology of solid tumors was the third-most common factor associated with IC/candidemia, after surgery and intensive care treatment [3]. Candida species are a frequent cause of invasive fungal infection in patients with many different types of solid tumors, including gastrointestinal, breast, ear/nose/throat, genitourinary, head and neck, hematologic, lung, liver, and skin [2, 4-8].

In epidemiology and surveillance studies, morbidity and mortality outcomes of IC in patients with solid tumors and hematologic cancers were poor $[3,4,9]$, with mortality rates between 30 and $65 \%$ [3-7, 9, 10]. In addition to older age and advanced disease, surgery, admission to the intensive care unit (ICU), and invasive procedures were factors associated with 30-day mortality $[5,7,9,10]$. Furthermore, $C$. albicans is reported as the most frequent isolate in patients with solid tumors followed by $C$. parapsilosis, $C$. tropicalis, or C. glabrata [3, 4, 6, 9, 11].

Appropriate use of antifungal treatment in patients with cancer and candidemia is a life-saving measure $[4,5,7,9]$. Echinocandins are an effective treatment against a range of Candida species [12], and the Infectious Diseases Society of America guidelines recommend echinocandins for treatment of IC/candidemia [13]. Also, guidelines from the European Society for Clinical Microbiology and Infectious Diseases strongly recommend echinocandins for the initial treatment of candidemia in adults [14].

\subsection{Study Data}

In this post hoc analysis, patient-level data were pooled and analyzed from four open-label, non-comparative studies of anidulafungin [20-23] and two double-blind, doubledummy, randomized studies (Pfizer data on file) that evaluated anidulafungin and caspofungin (Online Resource 1); all studies had similar protocols and endpoints. Data from all the above studies have been included in a previous analysis of the efficacy of anidulafungin in patients with IC [18], and this analysis followed a similar methodology.

Conduct was in accordance with applicable legal and regulatory requirements, the International Conference on Harmonization Guidelines for Good Clinical Practice, and the Declaration of Helsinki. The Institutional Review Boards or Independent Ethics Committees at each investigational center approved the studies. All patients provided written informed consent.

\subsection{Patients}

Male or female patients aged $\geq 18$ years for the four openlabeled and the two randomized studies were included in the pooled dataset if they had blood culture-confirmed candidemia, or IC confirmed by culture positive for Candida species isolated from a normally sterile site or a newly placed drain, with or without a positive Candida blood culture, within $96 \mathrm{~h}$ of entry to the study. Patients could be included based on microbiologic evidence of Candida infection, such as a positive blood (or tissue specimen) culture for yeast, provided confirmation of Candida species was obtained within $96 \mathrm{~h}$. 
Patients were also required to have one or more clinical signs and symptoms of fungal infection, including fever, hypothermia, hypotension, localized signs and symptoms of inflammation at a site with Candida infection within 48 $\mathrm{h}$ of commencing treatment, or radiologic findings indicative of IC. Patients were categorized as neutropenic if they had a baseline absolute neutrophil count (ANC) of $\leq 500$ cells $/ \mathrm{mm}^{3}$ (cells $\left./ \mu \mathrm{L}\right)$. Patients included in the analysis had either a past diagnosis of solid tumors (time of diagnosis $\geq 6$ months prior to study entry) or a recent diagnosis of solid tumors (time of diagnosis $<6$ months before study entry). Solid tumor status was obtained by examining the medical histories of patients using the following search criteria: tumor, neopla* (neoplastic, neoplasia, neoplasm), mass, cancer, malign* (malignant, malignancy), growth, oncol* (oncology, oncological), carcinoma, adenocarcinoma, and hepatoma.

The main exclusion criteria were: more than $48 \mathrm{~h}$ of systemic antifungal treatment prior to study entry; previous treatment failure for current episode of IC or candidemia; or a prosthetic device at a suspected site of infection that could not be removed prior to or within $24-48$ h of the start of the study.

\subsection{Treatment}

Patients received a single IV loading dose of anidulafungin $200 \mathrm{mg}$ on day 1 , followed by $100 \mathrm{mg}$ once daily and, in all studies except one [18], could be switched to oral voriconazole or fluconazole therapy after $\geq 5$ or $\geq 10$ days based on pre-specified criteria. IV anidulafungin and oral azole (if required) were maintained for $\geq 14$ days (and up to a maximum of 42 days) after the last positive Candida culture and resolution of symptoms.

\subsection{Endpoints}

The primary efficacy endpoint of this post hoc analysis was global response success (GRS) rate at the end of intravenous therapy (EOIVT) in the modified intent-to-treat (mITT) population. This included patients with solid tumors who received at least one dose of anidulafungin (the ITT population) and had a confirmed diagnosis of candidemia or a positive culture for Candida species at study commencement or within 96 h of study entry. GRS was achieved with clinical success, defined as cure or improvement of clinical signs and symptoms with no additional systemic or oral antifungal therapy, in conjunction with microbiologic success, defined as eradication or presumed eradication of baseline Candida species.

Secondary endpoints included GRS rate by baseline pathogen and site of infection, evaluated at EOIVT and end of all therapy (EOT) in the mITT population. Other secondary endpoints were all-cause mortality at days 14 and 28, duration of therapy (IV and IV + oral), and time to switch to oral azole therapy. Safety was assessed by the incidence and severity of adverse events occurring during or within 30 days of the last dose of treatment, recorded by system organ class and Medical Dictionary for Regulatory Activities (MedDRA) preferred terms. Safety analyses were based on the ITT population.

\subsection{Statistical Analysis}

All primary and secondary endpoints were summarized by the time of diagnosis. The past diagnosis was $\geq 6$ months since the time of diagnosis, and recent was $<6$ months. Analyses for baseline demographics and disease characteristics were for descriptive purposes only. Evaluations were primarily descriptive statistics. GRS rates were estimated with exact $95 \%$ confidence intervals (CI) for binomial proportion (Clopper-Pearson method), and 95\% CIs differences between past and recent diagnosis groups were estimated with exact unconditional limits. Comparisons between past and recent diagnosis groups were determined using a Fisher's exact test. Indeterminate or missing data were considered to be failures.

\section{Results}

\subsection{Patients}

Of 539 patients in the cohort pooled from six studies (Online Resource 1) [18], 150 had confirmed IC and history of any malignancy, and of these, 139 had solid tumors. Among patients with solid tumors (Table 1), baseline demographics and disease characteristics were generally comparable between the two diagnostic subgroups. The most frequent types of solid tumors were gastrointestinal (60.4\%), genitourinary $(24.5 \%)$, and lung (16.5\%). Metastases were present in $12.9 \%$ of all patients in the mITT population. Approximately one-half of the patients $(52.5 \%)$ were male and, overall, the mean age (standard deviation [SD]) was 63.1 (13.4) years. The majority of patients $(90.6 \%)$ were aged $\geq 45$ years, and $48.9 \%$ were aged $\geq 65$ years.

The overall mean (SD) Acute Physiology, Age, Chronic Health Evaluation (APACHE) II Score was 14.9 (5.5), and most patients $(90.9 \%)$ were non-neutropenic (Table 1$)$. The most frequent baseline pathogens were C. albicans $(48.9 \%)$ and $C$. glabrata $(21.6 \%)$. The main sites of infection were blood only $(75.5 \%)$, and other sterile sites $(20.1 \%)$, followed by blood and other sterile sites (4.3\%) [data not shown].

Frequent risk factors for IC included the use of broadspectrum antibiotics $(88.5 \%)$, central venous catheters $(80.3 \%)$, and total parenteral nutrition $(54.1 \%)$ (Table 1). 
The median (range) duration of IV + oral therapy for the mITT population was 15.0 (1-56) days. Fifty-four of 139 patients $(38.8 \%)$ in the mITT population switched to oralonly therapy for a median (range) duration of 9.0 (5-35) days, and a median time to switch of 8.0 days (range: 4-34). Among these patients, the proportion of patients switching to oral azoles was lower in patients with a past diagnosis of solid tumors than a recent diagnosis $(42.6 \%$ vs $57.4 \%$, respectively), and the median time to switch was shorter (6 vs 10 days, respectively).

\subsection{Efficacy Endpoints}

The GRS rate in the mITT population was $73.4 \%(102 / 139)$ at EOIVT (primary endpoint) and 65.5\% (91/139) at EOT (Fig. 1). GRS rates at EOIVT and EOT in patients with a past tumor diagnosis were 75.5\% (37/49) and 71.4\% (35/49); corresponding GRS rates were $72.2 \%$ (65/90) and $62.2 \%$ (56/90), respectively, in those with a recent diagnosis of solid tumors (Fig. 1). GRS rates at both EOIVT and EOT were not significantly different between those with recent or past diagnosis of solid tumors $(p>0.05)$.

GRS rates at EOIVT and EOT in the overall population were $77.9 \%$ and $69.1 \%$, respectively, for C. albicans, and $67.1 \%$ and $61.2 \%$, respectively, for non-albicans Candida species (Table 2). GRS rate by the site of infection was $75.2 \%$ for blood-only infections at EOIVT and $65.7 \%$ at EOT (Table 2). For patients with infection in the blood only, those with recent solid tumor diagnosis had a significantly smaller GRS at EOT, compared with patients with past solid tumor diagnoses $(p=0.049)$.

All-cause mortality in the overall mITT population was $20 / 139(14.4 \%)$ at day 14 and 28/139 (20.1\%) at day 28. Allcause mortality rates were not significantly different between populations with a recent and past diagnosis of solid tumors at day 14 or overall. At day 28, the all-cause mortality rate was significantly higher in those with a recent diagnosis of solid tumors (25.6\%), compared with those with a past diagnosis $(10.2 \%)(p=0.045)$.

\subsection{Safety Endpoints}

Incidence and severity of treatment-emergent adverse events (TEAEs) are shown in Table 3. A total of 787 TEAEs were observed in 123 patients, and the proportion of patients with events was similar in those with a recent $(88.9 \%)$ or past diagnosis of solid tumors (87.8\%) (data not shown). The majority of all-causality TEAEs in the overall population were mild or moderate in severity $(642 / 787 ; 81.6 \%)$. The most common TEAEs were gastrointestinal, including diarrhea $(21 / 139 ; 15.1 \%)$, nausea $(13 / 139 ; 9.4 \%)$, and vomiting $(13 / 139 ; 9.4 \%)$; the pattern was similar in the two tumor subgroups (data not shown).

\section{Discussion}

This analysis of patient-level data pooled from six prospective studies of anidulafungin showed that in patients with IC/candidemia and solid tumors, the GRS rate at EOIVT (73.4\%; primary endpoint) was comparable to the overall GRS rate (75.6\% at EOIVT) in patients with IC in the registrational study [24]. The GRS rates were also comparable to a randomized, double-blind comparison of caspofungin and amphotericin B in 74 patients with IC and active malignancies. In that investigation, response rates in patients with solid tumors were $80 \%$ and $59 \%$ in the two treatment arms, respectively [2].

Additionally, in patients with solid tumors, all-cause mortality rate by $6-8$ weeks following discontinuation of IV treatment was $40 \%$ in caspofungin recipients and $21 \%$ in recipients of amphotericin B [2]. By comparison, the present analysis included a higher number of patients with IC/ candidemia and solid tumors, and the all-cause mortality rate was $20.1 \%$ at day 28 .

Non-albicans Candida infection also represents concern in daily clinical practice [1]. In our analysis, C. albicans was overall the most common isolate, and GRS rates were slightly lower in patients with baseline $C$. albicans and a past solid tumor diagnosis compared with a recent diagnosis at EOIVT (74\% vs $80 \%)$, but not at EOT (70\% vs $69 \%$ ). Overall, anidulafungin was effective in patients with baseline $C$. albicans and non-albicans Candida infection (78\% and 67\% at EOIVT, respectively). The characteristics of patients in our analysis were generally consistent with other studies $[4$, $6,9]$, including $49 \%$ in our mITT population with IC/candidemia due to $C$. albicans, $60 \%$ with solid tumors that were located in the gastrointestinal tract, and around half (53\%) having undergone abdominal surgery. In a separate pooled analysis of 79 patients with intra-abdominal candidiasis, the overall global response rate following anidulafungin treatment was $73.4 \%$ at EOIVT [19].

Neutropenia is a more common characteristic of patients with hematologic cancer than patients with solid tumors [9]. Our population was comparable with this observation in that the majority with solid tumors were non-neutropenic at baseline (91\%).

There is a paucity of studies that have evaluated the time of solid tumor diagnosis as a factor that may determine the clinical outcome, and in our analysis, the GRS rate was comparable at EOIVT regardless of past or recent diagnosis of solid tumors. At EOT, the pattern of global response rates was comparable. In a retrospective cohort study of 144 non-neutropenic patients with candidemia, multivariate analysis showed that one of the predictors of non-albicans candidemia was a recent history of solid tumor [25]. A prospective, multicenter, surveillance program in Spain, 
Table 1 Patient baseline demographics and disease characteristics by time of solid tumor diagnosis $(\mathrm{mITT}, N=139)$

\begin{tabular}{|c|c|c|c|}
\hline \multirow[t]{2}{*}{ Characteristic } & \multicolumn{2}{|c|}{ Time of solid tumor diagnosis ${ }^{\mathrm{a}}$} & \multirow[t]{2}{*}{ Total } \\
\hline & Past $(\geq 6 \mathrm{~m})$ & Recent $(<6 \mathrm{~m})$ & \\
\hline Number $(\%)$ of patients & $49(35.3)$ & $90(64.7)$ & $139(100)$ \\
\hline Sex, $n(\%)$ male & $26(53.1)$ & $47(52.2)$ & $73(52.5)$ \\
\hline \multicolumn{4}{|l|}{ Patients in age group, $\mathrm{n}(\%)$} \\
\hline $18-44$ years & $3(6.1)$ & $10(11.1)$ & $13(9.4)$ \\
\hline $45-64$ years & $20(40.8)$ & $38(42.2)$ & $58(41.7)$ \\
\hline$\geq 65$ years & $26(53.1)$ & $42(46.7)$ & $68(48.9)$ \\
\hline Age, mean (SD) years & $65.9(12.5)$ & $61.6(13.7)$ & $63.1(13.4)$ \\
\hline \multicolumn{4}{|l|}{ Race, $n(\%)$} \\
\hline White & 38 (77.6) & $56(62.2)$ & $94(67.6)$ \\
\hline Black & $5(10.2)$ & $10(11)$ & $15(10.8)$ \\
\hline Asian & $2(4.1)$ & $21(23.3)$ & $23(16.5)$ \\
\hline Other & $4(8.2)^{b}$ & $3(3.3)$ & $7(5.0)$ \\
\hline Weight, mean (SD) kg & $75.3(20.1)$ & $68.0(16.9)^{\mathrm{c}}$ & $70.6(18.3)$ \\
\hline Height, mean (SD) cm & $168.5(9.0)^{\mathrm{d}}$ & $167.3(10.4)^{\mathrm{e}}$ & $167.7(9.9)$ \\
\hline APACHE II score, mean (SD) & $14.4(5.3)$ & $15.1(5.7)^{\mathrm{c}}$ & $14.9(5.5)$ \\
\hline \multicolumn{4}{|l|}{$n(\%)$ patients with a score ${ }^{\mathrm{f}}$} \\
\hline$\leq 20$ & $42(85.7)$ & $73(81.1)$ & $115(82.7)$ \\
\hline$>20$ & $7(14.3)$ & $16(17.8)$ & $23(16.5)$ \\
\hline ANC, ${ }^{\mathrm{g}}$ mean $(\mathrm{SD}) 10^{3} / \mathrm{mm}^{3}$ & $6.3(5.0)^{\mathrm{h}}$ & $7.2(5.7)^{\mathrm{i}}$ & $6.9(5.5)$ \\
\hline \multicolumn{4}{|l|}{$n(\%)$ patients with } \\
\hline$\leq 500$ cells $/ \mathrm{mm}^{3}$ & $3(6.1)$ & $5(5.6)$ & $8(5.8)$ \\
\hline$>500$ cells $/ \mathrm{mm}^{3}$ & $28(57.1)$ & $52(57.8)$ & $80(57.6)$ \\
\hline \multicolumn{4}{|l|}{ Baseline diagnosis, $n(\%)$} \\
\hline Systemic Candida ${ }^{\mathrm{j}}$ & $48(98.0)$ & $88(97.8)$ & $136(97.8)$ \\
\hline Candidal sepsis & $1(2.0)$ & $1(1.1)$ & $2(1.4)$ \\
\hline Empyema & - & $1(1.1)$ & $1(0.7)$ \\
\hline \multicolumn{4}{|l|}{ Type of solid tumor, $n(\%)$} \\
\hline Breast & $3(6.1)$ & $7(7.8)$ & $10(7.2)$ \\
\hline Gastrointestinal & $24(49.0)$ & $60(66.7)$ & $84(60.4)$ \\
\hline Genitourinary & 14 (28.6) & $20(22.2)$ & $34(24.5)$ \\
\hline Head/neck & $2(4.1)$ & $4(4.4 \%)$ & $6(4.3 \%)$ \\
\hline Lung & $6(12.2)$ & $17(18.9)$ & $23(16.5)$ \\
\hline Skin & $3(6.1)$ & $2(2.2)$ & $5(3.6)$ \\
\hline Other/missing & $12(24.5)$ & $15(16.7)$ & $27(19.4)$ \\
\hline Patients with known metastases, $n(\%)^{\mathrm{k}}$ & $5(10.2)$ & $13(14.4)$ & $18(12.9)$ \\
\hline \multicolumn{4}{|l|}{ Baseline pathogen, $n(\%)^{1}$} \\
\hline C. albicans & $23(46.9)$ & $45(50.0)$ & $68(48.9)$ \\
\hline C. glabrata & $14(28.6)$ & $16(17.8)$ & $30(21.6)$ \\
\hline C. parapsilosis & $8(16.3)$ & $12(13.3)$ & $20(14.4)$ \\
\hline C. tropicalis & $6(12.2)$ & $16(17.8)$ & $22(15.8)$ \\
\hline C. kefyr & $2(4.1)$ & - & $2(1.4)$ \\
\hline C. krusei & $2(4.1)$ & - & $2(1.4)$ \\
\hline C. lusitaniae & $1(2.0)$ & - & $1(0.7)$ \\
\hline C. famata & - & $2(2.2)$ & $2(1.4)$ \\
\hline C. sake & - & $1(1.1)$ & $1(0.7)$ \\
\hline Candida spp. & - & $1(1.1)$ & $1(0.7)$ \\
\hline Other & - & $4(4.4)$ & $4(2.9)$ \\
\hline
\end{tabular}


Table 1 (continued)

\begin{tabular}{|c|c|c|c|}
\hline \multirow[t]{2}{*}{ Characteristic } & \multicolumn{2}{|c|}{ Time of solid tumor diagnosis ${ }^{\mathrm{a}}$} & \multirow[t]{2}{*}{ Total } \\
\hline & Past $(\geq 6 \mathrm{~m})$ & Recent $(<6 \mathrm{~m})$ & \\
\hline \multicolumn{4}{|l|}{ Risk factors for invasive candidiasis, $n(\%)^{\mathrm{m}}$} \\
\hline Use of broad-spectrum antibiotics & $43(91.5)$ & $65(86.7)$ & $108(88.5)$ \\
\hline Use of central venous catheter & $40(85.1)$ & $58(77.3)$ & $98(80.3)$ \\
\hline Total parenteral nutrition & $24(51.1)$ & $42(56.0)$ & $66(54.1)$ \\
\hline Abdominal surgery & $27(57.4)$ & $37(49.3)$ & $64(52.5)$ \\
\hline Surgery & $23(48.9)$ & $39(52.0)$ & $62(50.8)$ \\
\hline Length of ICU stay $\geq 4$ days & $17(36.2)$ & $26(34.7)$ & $43(35.2)$ \\
\hline Mechanical ventilation & $14(29.8)$ & $24(32.0)$ & $38(31.1)$ \\
\hline Chemotherapy & $13(27.7)$ & $18(24.0)$ & $31(25.4)$ \\
\hline Use of systemic steroids/other immunosuppressants & $12(25.5)$ & $19(25.3)$ & $31(25.4)$ \\
\hline Renal insufficiency/failure/dialysis & $10(21.3)$ & $15(20.0)$ & $25(20.5)$ \\
\hline Neutropenia & $3(6.4)$ & $7(9.3)$ & $10(8.2)$ \\
\hline Solid organ transplant & $3(6.4)$ & 0 & $3(2.5)$ \\
\hline Other & 15 (31.9) & $19(25.3)$ & 34 (27.9) \\
\hline
\end{tabular}

APACHE Acute Physiology, Age, Chronic Health Evaluation, ANC absolute neutrophil count, ICU intensive care unit, $m$ months

a Past diagnosis of solid tumors, $\geq 6$ months prior to study entry; recent diagnosis, $<6$ months before study entry

${ }^{\mathrm{b}} 2$ other, and 2 unspecified

${ }^{\mathrm{c}} N=89$

${ }^{\mathrm{d}} N=48$

${ }^{\mathrm{e}} N=85$

${ }^{\mathrm{f}} N=138$

${ }^{\mathrm{g}}$ Number of patients for whom ANC was recorded at baseline

${ }^{\mathrm{h}} N=31$

${ }^{\mathrm{i}} \mathrm{N}=57$

${ }^{j}$ Systemic Candida included candidiasis, peritoneal candidiasis, fungal peritonitis, blood culture positive, biliary tract infection fungal, fungemia, and fungal test positive

${ }^{\mathrm{k}}$ Metastases recorded as 'Yes/No' (Yes = known metastases)

${ }^{\text {l} P a t i e n t s ~ c o u l d ~ h a v e ~ m o r e ~ t h a n ~ o n e ~ C a n d i d a ~ s p e c i e s ~ i s o l a t e d ~ a t ~ b a s e l i n e ~}$

${ }^{\mathrm{m}}$ Patients could have more than one risk factor at baseline

conducted in part to identify predictors of death in candidiasis, found that 7 - versus 30-day mortality in patients with hematologic malignancies and solid tumors was $12.8 \%(93 / 726)$ and $30.6 \%$ (220/720). When adjusted by the primary source of candidemia and severity of infection (severe sepsis or septic shock) in the multivariate regression analysis, appropriate antifungal treatment within the first $48 \mathrm{~h}$ was the only factor independently associated with lower mortality [26]. This highlights the importance of early antifungal treatment of candidiasis in patients with solid organ tumors.
De-escalation of antifungal treatment has been assessed in a study of 190 critically ill patients with suspected IC [27]. The approach, which was undertaken in $20 \%$ of the patients included, was considered to be safe and was associated with a shorter median duration of antifungal treatment compared with no de-escalation (6 vs 13 days). In our study, a higher proportion of patients (38.8\%) were switched to oral therapy, and the median total duration of therapy (IV + oral) was similar in past and recent solid tumor diagnostic sub-groups (15 vs 14 days, respectively). 
Fig. 1 Anidulafungin global response success rates at EOIVT and EOT by the time of solid tumor diagnosis (mITT, $N=139)$. EOIVT end of intravenous treatment; EOT end of treatment; $m$ months; $n s$ not significant based on Fisher's exact test. Past diagnosis of solid tumors, $\geq 6$ months prior to study entry; recent diagnosis, $<6$ months before study entry
Past $(\geq 6 \mathrm{~m}) \quad$ Recent $(<6 \mathrm{~m}) \quad$ Total

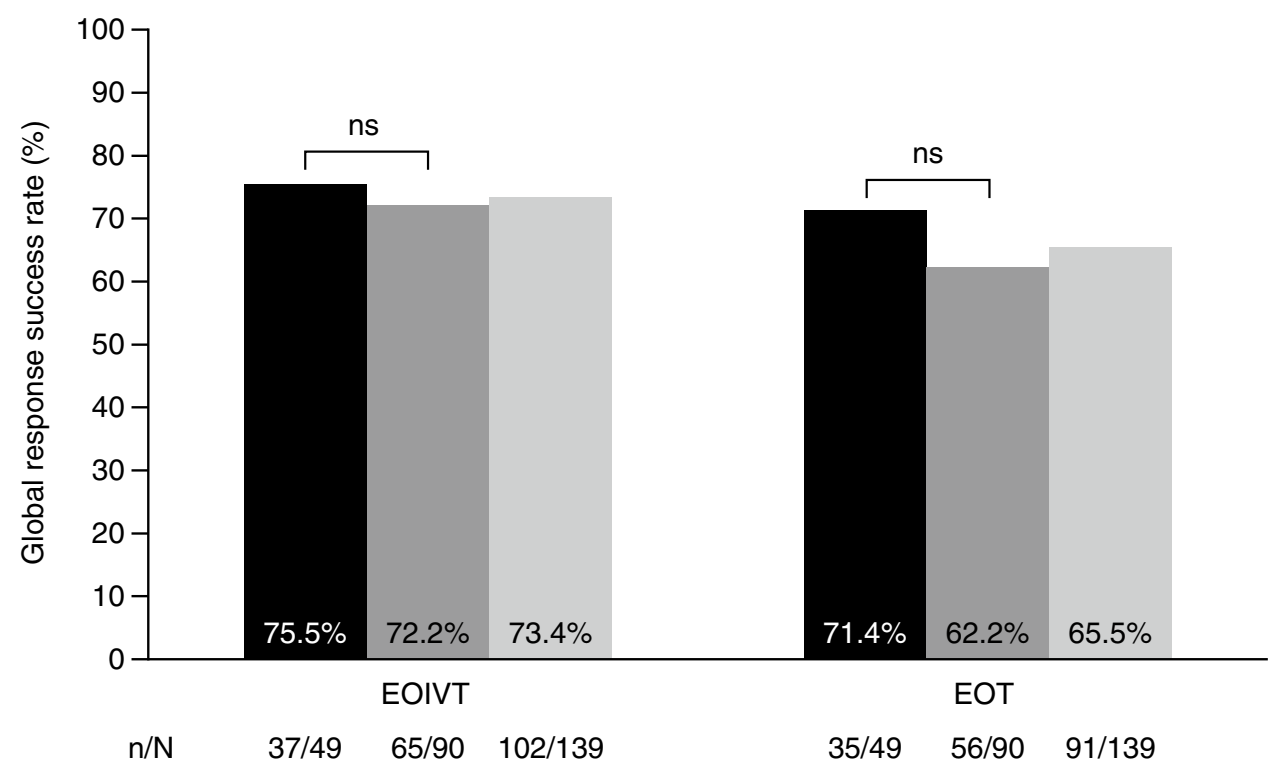

Elderly patients (aged $\geq 65$ years) formed almost half $(48.9 \%)$ of the patients in this post hoc analysis, and older age is a factor associated with IC/candidemia in patients with cancer [9]. In another post hoc analysis of anidulafungin treatment in critically ill patients, a similar proportion (47\%) were elderly patients with IC/candidemia, and the median duration of IV anidulafungin treatment was comparable in elderly and non-elderly patients (14.0 vs 14.5 days) [28], similar to the present analysis. Overall, switch to oral fluconazole or voriconazole occurred in 58/170 (34\%) of patients in the earlier investigation [28], also similar to the proportion in our analysis.

Overall, anidulafungin was well tolerated, and the majority of TEAEs were mild-to-moderate in severity; no new safety signals arose from this analysis [24]. The number of TEAEs experienced by patients with a past or recent diagnosis of solid tumors was generally similar.

One limitation of this analysis was that four studies were open-label and conducted at different times. However, all studies had a similar design that permitted the pooling of data. Our analysis was post hoc and not pre-planned by the individual study protocols. Susceptibility testing was not evaluated as part of this pooled analysis but has been described for a number of the individual prospective studies of anidulafungin [20,22, 23, 28], and in pooled populations [17, 18]. Also, due to the limited number of patients, no multivariate analysis was performed, and patients were included either with solid tumors or a history of solid tumors. Selection of the time of past or present diagnosis of solid tumors did not lead to the identification of a difference between the effect of treatment in the two sub-groups, but we cannot exclude the possibility that further investigations using this or a different definition and a larger group of patients may be needed. Our study did not evaluate the potential for drug-drug interactions (DDIs). In recent years, several new immunotherapeutic agents have been developed for the treatment of patients with hematologic malignancies and solid tumors, and, for some of these new agents, the use of azoles that are strong inhibitors of cytochrome $\mathrm{P} 4503 \mathrm{~A} 4$ is precluded due to the potential for DDIs [29]. Anidulafungin does not undergo hepatic metabolism, nor does it interact with the cytochrome P450 enzymes involved in DDIs [30]. No significant interactions between anidulafungin and cyclosporine, tacrolimus, rifampin, voriconazole, or liposomal amphotericin B are currently known [16].

\section{Conclusion}

Anidulafungin was effective for the treatment of IC/candidemia in patients regardless of past or recent time of solid tumor diagnosis. The safety results were consistent with the known profile of anidulafungin. 


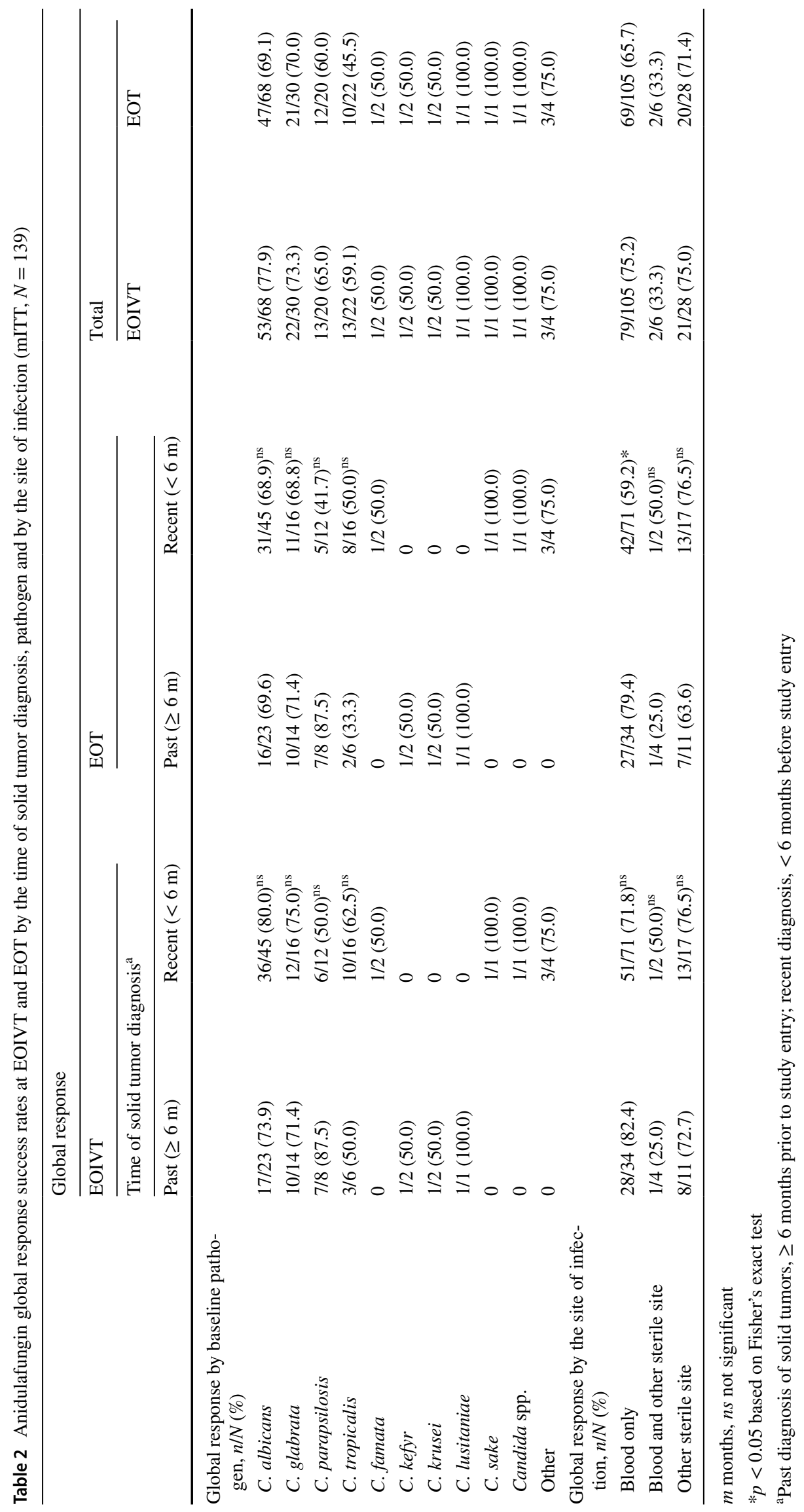


Table 3 Incidence and severity of treatment-emergent adverse events during all treatment (IV anidulafungin + oral azole) by system organ class (ITT)

\begin{tabular}{lll}
\hline & \multicolumn{2}{l}{ Total } \\
\cline { 2 - 3 } Patients with adverse events, $n / N(\%)$ & $123 / 139(88.5)$ \\
\cline { 2 - 3 } Total preferred term events, $N$ & 787 \\
Severity: ${ }^{\mathrm{a}}$ mild/moderate/severe & $350 / 292 / 145$ \\
\cline { 2 - 3 } Category & Events & Severe \\
& $($ any sever- & events, \\
& ity) & $n$ \\
\hline Gastrointestinal & $n(\%)$ & \\
Infections and infestations & $64(46.0)$ & 12 \\
General and administration site conditions & $62(44.6)$ & 22 \\
Respiratory, thoracic and mediastinal & $47(33.8)$ & 14 \\
Metabolism and nutrition & $46(33.1)$ & 18 \\
Psychiatric & $44(31.7)$ & 3 \\
Vascular & $33(23.7)$ & 2 \\
Investigations & $31(22.3)$ & 9 \\
Cardiac & $29(20.9)$ & 1 \\
Blood and lymphatic system & $28(20.1)$ & 9 \\
Renal and urinary tract & $26(18.7)$ & 7 \\
Nervous system & $20(14.4)$ & 6 \\
Skin and subcutaneous tissue & $20(14.4)$ & 3 \\
Neoplasms, benign, malignant and unspecified & $15(10.8)$ & 13 \\
Injury, poisoning and procedural complications & $13(9.4)$ & 3 \\
Musculoskeletal and connective tissue & $12(8.6)$ & 0 \\
Hepatobiliary & $11(7.9)$ & 3 \\
Eye & $7(5.0)$ & 0 \\
Product issues ${ }^{c}$ & $6(4.3)$ & 0 \\
Surgical and medical & $4(2.9)$ & 1 \\
Reproductive system and breast & $2(1.4)$ & 1 \\
Immune system & $2(1.4)$ & 0 \\
Ear and labyrinth & $2(1.4)$ & 0 \\
\hline & & 2 \\
\hline
\end{tabular}

$I V$ intravenous, $m$ months, MedDRA Medical Dictionary for Regulatory Activities

${ }^{a}$ If the same patient in a given treatment had more than one occurrence in the same preferred term event category, only the most severe occurrence is taken. Patients are counted only once per treatment in each row

${ }^{\mathrm{b}}$ MedDRA v19.1

${ }^{\mathrm{c}}$ Includes device breakage, device occlusion

Acknowledgements Data from this analysis were presented at the 27th European Congress of Clinical Microbiology and Infectious Diseases (ECCMID), Vienna, Austria, April 22-25, 2017. Editorial/medical writing support, under the direction of the authors, was provided by Neil Cockburn, BSc, and Emma Mitchell, Ph.D., CMC Connect, McCann Health Medical Communications, and was funded by Pfizer Inc, New York, NY, USA in accordance with Good Publication Practice (GPP3) guidelines (Ann Intern Med 2015;163:461-464).
Author contributions All authors were involved in data analysis and interpretation, manuscript writing, and reviewed and approved the manuscript final draft for submission. FDR was involved in study methodology, data analysis discussions, data interpretation, drafting, and reviewing the manuscript. $\mathrm{AB}$ was involved in critically reviewing the manuscript. JAA, JLY, and MRC were involved in the analysis concept and data collection.

\section{Declarations}

Funding Open access funding provided by Università degli Studi di Torino within the CRUI-CARE Agreement. This study was sponsored by Pfizer Inc.

Conflict of interest FDR has received speaker grants and participated in advisory boards for Angelini, Basilea, Biomereuix, BioTest, Correvio, Gilead Sciences, Hikma, MSD, Nordic Pharma, Sanofi Aventis, Pfizer Inc, and ThermoFisher. AB has received honoraria from Gilead Sciences, Jazz Pharmaceuticals, Merck, and Pfizer Inc; he has been speaker for Gilead Sciences, Merck, Novartis, and Pfizer Inc, and has served on Advisory Boards for Gilead Sciences and Pfizer Inc. JAA, JLY, and MRC are employees and shareholders of Pfizer Inc.

Ethical approval Conduct was in accordance with applicable legal and regulatory requirements, the International Conference on Harmonization Guidelines for Good Clinical Practice, and the Declaration of Helsinki. The Institutional Review Boards or Independent Ethics Committees at each investigational center approved the studies.

Consent to participate All patients provided written informed consent.

Availability of data and material Upon request, and subject to certain criteria, conditions, and exceptions (see https://www.pfizer.com/scien ce/clinical-trials/trial-data-and-results for more information), Pfizer will provide access to individual de-identified participant data from Pfizer-sponsored global interventional clinical studies conducted for medicines, vaccines, and medical devices (1) for indications that have been approved in the US and/or EU or (2) in programs that have been terminated (i.e. development for all indications has been discontinued). Pfizer will also consider requests for the protocol, data dictionary, and statistical analysis plan. Data may be requested from Pfizer trials 24 months after study completion. The de-identified participant data will be made available to researchers whose proposals meet the research criteria and other conditions, and for which an exception does not apply, via a secure portal. To gain access, data requestors must enter into a data access agreement with Pfizer.

Open Access This article is licensed under a Creative Commons Attribution-NonCommercial 4.0 International License, which permits any non-commercial use, sharing, adaptation, distribution and reproduction in any medium or format, as long as you give appropriate credit to the original author(s) and the source, provide a link to the Creative Commons licence, and indicate if changes were made. The images or other third party material in this article are included in the article's Creative Commons licence, unless indicated otherwise in a credit line to the material. If material is not included in the article's Creative Commons licence and your intended use is not permitted by statutory regulation or exceeds the permitted use, you will need to obtain permission directly from the copyright holder. To view a copy of this licence, visit http://creativecommons.org/licenses/by-nc/4.0/. 


\section{References}

1. Yapar N. Epidemiology and risk factors for invasive candidiasis. Ther Clin Risk Manag. 2014;10:95-105.

2. DiNubile MJ, Hille D, Sable CA, Kartsonis NA. Invasive candidiasis in cancer patients: observations from a randomized clinical trial. J Infect. 2005;50:443-9.

3. Tortorano AM, Kibbler C, Peman J, Bernhardt H, Klingspor L, Grillot R. Candidaemia in Europe: epidemiology and resistance. Int J Antimicrob Agents. 2006;27:359-66.

4. Tang HJ, Liu WL, Lin HL, Lai CC. Epidemiology and prognostic factors of candidemia in cancer patients. PLoS ONE. 2014;9:e99103.

5. Lortholary O, Renaudat C, Sitbon K, Desnos-Ollivier M, Bretagne $\mathrm{S}$, Dromer F, et al. The risk and clinical outcome of candidemia depending on underlying malignancy. Intensive Care Med. 2017;43:652-62.

6. Pasqualotto AC, Rosa DD, Medeiros LR, Severo LC. Candidaemia and cancer: patients are not all the same. BMC Infect Dis. 2006;6:50

7. Viscoli C, Girmenia C, Marinus A, Collette L, Martino P, Vandercam B, et al. Candidemia in cancer patients: a prospective, multicenter surveillance study by the Invasive Fungal Infection Group (IFIG) of the European Organization for Research and Treatment of Cancer (EORTC). Clin Infect Dis. 1999;28:1071-9.

8. Ghrenassia E, Mokart D, Mayaux J, Demoule A, Rezine I, Kerhuel L, et al. Candidemia in critically ill immunocompromised patients: report of a retrospective multicenter cohort study. Ann Intensive Care. 2019;9:62.

9. Puig-Asensio M, Ruiz-Camps I, Fernandez-Ruiz M, Aguado JM, Munoz P, Valerio M, et al. Epidemiology and outcome of candidaemia in patients with oncological and haematological malignancies: results from a population-based surveillance in Spain. Clin Microbiol Infect. 2015;21(491):e1-10.

10. Bergamasco MD, Garnica M, Colombo AL, Nucci M. Epidemiology of candidemia in patients with hematologic malignancies and solid tumours in Brazil. Mycoses. 2013;56:256-63.

11. Sun M, Chen C, Xiao W, Chang Y, Liu C, Xu Q. Increase in candida parapsilosis candidemia in cancer patients. Mediterr $\mathrm{J}$ Hematol Infect Dis. 2019;11:e2019012.

12. Sucher AJ, Chahine EB, Balcer HE. Echinocandins: the newest class of antifungals. Ann Pharmacother. 2009;43:1647-57.

13. Pappas PG, Kauffman CA, Andes DR, Clancy CJ, Marr KA, Ostrosky-Zeichner L, et al. Clinical practice guideline for the management of candidiasis: 2016 update by the Infectious Diseases Society of America. Clin Infect Dis. 2016;62:e1-50.

14. Cornely OA, Bassetti M, Calandra T, Garbino J, Kullberg BJ, Lortholary O, et al. ESCMID* guideline for the diagnosis and management of Candida diseases 2012: non-neutropenic adult patients. Clin Microbiol Infect. 2012;18(Suppl. 7):19-37.

15. European Medicines Agency. ECALTA summary of product characteristics. 2016. https://www.medicines.org.uk/emc/medic ine/22690. Accessed 2 Mar 2020.

16. Pfizer. Eraxis highlights of prescribing information. 2019. http:// labeling.pfizer.com/ShowLabeling. aspx ?format=PDF\&id $=566$. Accessed 2 Mar 2020
17. Kontoyiannis DP, Bassetti M, Nucci M, Capparella MR, Yan JL, Aram J, et al. Anidulafungin for the treatment of candidaemia caused by Candida parapsilosis: analysis of pooled data from six prospective clinical studies. Mycoses. 2017;60:663-7.

18. Kullberg BJ, Vasquez J, Mootsikapun P, Nucci M, Paiva JA, Garbino J, et al. Efficacy of anidulafungin in 539 patients with invasive candidiasis: a patient-level pooled analysis of six clinical trials. J Antimicrob Chemother. 2017;72:2368-77.

19. Sganga G. Evaluation of anidulafungin in the treatment of intraabdominal candidiasis: a pooled analysis of patient-level data from 5 prospective studies. Eur J Clin Microbiol Infect Dis. 2019;38:1849-56.

20. Vazquez J, Reboli AC, Pappas PG, Patterson TF, Reinhardt J, Chin-Hong P, et al. Evaluation of an early step-down strategy from intravenous anidulafungin to oral azole therapy for the treatment of candidemia and other forms of invasive candidiasis: results from an open-label trial. BMC Infect Dis. 2014;14:97.

21. Nucci M, Colombo AL, Petti M, Magana M, Abreu P, Schlamm HT, et al. An open-label study of anidulafungin for the treatment of candidaemia/invasive candidiasis in Latin America. Mycoses. 2014;57:12-8.

22. Mootsikapun P, Hsueh PR, Talwar D, Co VM, Rajadhyaksha V, Ong ML. Intravenous anidulafungin followed optionally by oral voriconazole for the treatment of candidemia in Asian patients: results from an open-label phase III trial. BMC Infect Dis. 2013;13:219.

23. Ruhnke M, Paiva JA, Meersseman W, Pachl J, Grigoras I, Sganga $\mathrm{G}$, et al. Anidulafungin for the treatment of candidaemia/invasive candidiasis in selected critically ill patients. Clin Microbiol Infect. 2012;18:680-7.

24. Reboli AC, Rotstein C, Pappas PG, Chapman SW, Kett DH, Kumar D, et al. Anidulafungin versus fluconazole for invasive candidiasis. N Engl J Med. 2007;356:2472-82.

25. Davis SL, Vazquez JA, McKinnon PS. Epidemiology, risk factors, and outcomes of Candida albicans versus non-albicans candidemia in nonneutropenic patients. Ann Pharmacother. 2007;41:568-73.

26. Puig-Asensio M, Padilla B, Garnacho-Montero J, Zaragoza O, Aguado JM, Zaragoza R, et al. Epidemiology and predictive factors for early and late mortality in Candida bloodstream infections: a population-based surveillance in Spain. Clin Microbiol Infect. 2014;20:O245-54.

27. Jaffal K, Poissy J, Rouze A, Preau S, Sendid B, Cornu M, et al. De-escalation of antifungal treatment in critically ill patients with suspected invasive Candida infection: incidence, associated factors, and safety. Ann Intensive Care. 2018;8:49.

28. Dimopoulos G, Paiva JA, Meersseman W, Pachl J, Grigoras I, Sganga G, et al. Efficacy and safety of anidulafungin in elderly, critically ill patients with invasive Candida infections: a post hoc analysis. Int J Antimicrob Agents. 2012;40:521-6.

29. Campen CJ, Vogel WH, Shah PJ. Managing drug interactions in cancer therapy: a guide for the advanced practitioner. J Adv Pract Oncol. 2017;8:609-20.

30. Damle BD, Dowell JA, Walsky RL, Weber GL, Stogniew M, Inskeep PB. In vitro and in vivo studies to characterize the clearance mechanism and potential cytochrome $\mathrm{P} 450$ interactions of anidulafungin. Antimicrob Agents Chemother. 2009;53:1149-56. 